\title{
«Death Sentences»: \\ «Wie man wird, was man ist» \\ (Empedrando la «bêtise» en J. D.)
}

\author{
Federico RODRÍGUEZ GÓMEZ \\ Universidad de Sevilla \\ frogo@us.es
}

\section{Resumen}

Este artículo, sembrado de piedras y empedrado con diferentes lenguas, gira en torno a la «bêtise» [término francés que suele traducirse, no sin dejar restos, por tontería, bobada, etc.] en filosofía. A partir de la lectura de una serie de textos de Derrida, se destacan entre otros asuntos lo que el autor de este artículo denomina «dos cabos sueltos» de la reflexión sobre la «bêtise»: 1) la «bêtise» que consiste en querer concluir de una vez por todas en un intento de apropiación, lo cual es a su vez lo propio de la filosofía y, por consiguiente, también lo propio de ese animal racional que es el hombre; y 2) la relación que la «bêtise» mantiene con la bestialidad así como con la crueldad y con el mal.

Palabras clave: bêtise, bestialidad, lo propio del hombre, muerte, mal radical.

\begin{abstract}
This article, scattered with cobbles and cobbled with different languages, revolves around the bêtise [a French term that usually translates as "stupidity," "nonsense," etc.] in philosophy. Starting from the reading of a series of texts by Derrida, the author focuses, among other issues, on what he calls "two loose ends" of the reflection upon the betise: 1) the bêtise that consists in willing to conclude once and for all in an attempt to appropriate, which is in its turn proper to philosophy, and therefore proper to that rational animal that man is; and 2) the relationship that the bêtise maintains with bestiality, just as with cruelty and evil.
\end{abstract}

Key words: Bêtise, bestiality, the proper to man, death, radical evil. 


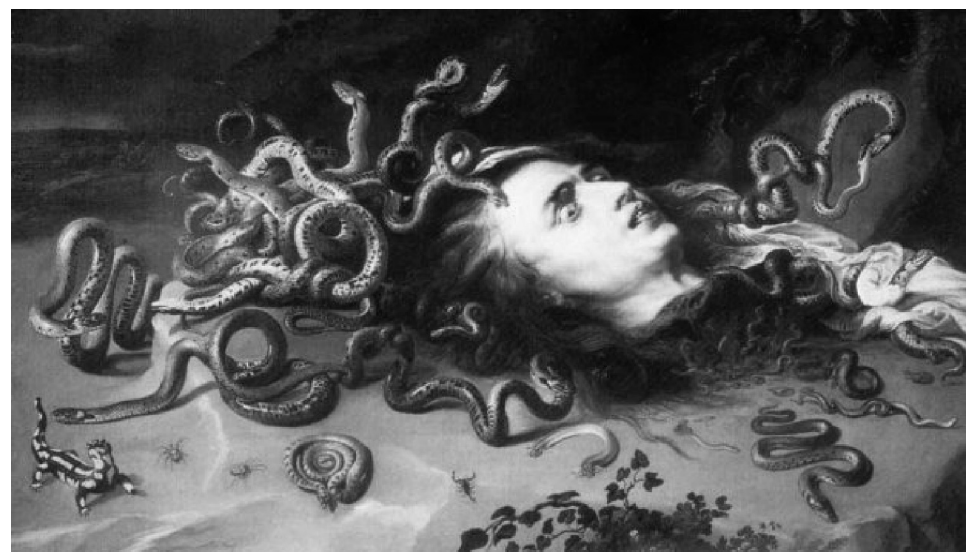

P. P. Rubens, Cabeza de Medusa, 1617

[decapitada a todas luces mediante todas las luces].

«Jenny: Are you stupid or something?

Forrest: Mommy says stupid is as stupid does».

(Forrest Gump, 1989)

«Je suis, pour mon compte, effrayé par la bêtise universelle! Cela me fait l'effet du déluge et j'éprouve la terreur que devaient subir les contemporaines de Noé, quand ils voyaient l'inondation envahir successivement tous les sommets. Les gens d'esprit devraient construire quelque chose d'analogue à l'Arche, s'y enfermer et vivre ensemble». (Flaubert, à la Princesse Mathilde, 8 juin 1874)

«Contagion de la bêtise»: debieran quedarse de piedra, con cara de tontos (méduser) ${ }^{1}$, sin mundo (weltlos) ${ }^{2}$. Dicho de otro modo, hubieran debido satirizar su abismo justo antes de desfallecer como desfallecen: despeñándose, rodando ladera abajo, de manera escandalosa $(\sigma \kappa a ́ v \delta \alpha \lambda o v)^{3}$. O al menos, si tuvieran algo de sensibilidad, cierta predisposición para el llanto y las lágrimas, para la empatía por poco patética ( $\pi \alpha \theta \eta \tau \imath \kappa o ́ \varsigma)$ que la misma fuera, lo cual es seguramente pedirles demasia-

\footnotetext{
${ }^{1}$ Derrida, J., «Parergon», en La vérité en peinture, Paris, Flammarion, 1978, p. 163.

2 Cfr., Heidegger, M., Die Grundbegriffe der Metaphysik. Welt-Endlichkeit-Einsamkeit, en Gesamtausgabe, II. Abteilung: Vorlesungen 1923-1944, vol. 49/30, Frankfurt am Main, 1992; Cfr., Derrida, J., De l'esprit. Heidegger et la question, Paris, Galilée, 1987, p. 21 y ss; Cfr., Apories. Mourir-s'attendre aux limites de la vérité, Paris, Galilée, 1996, p. 132; Cfr., L'animal que donc je suis, Paris, Galilée, 2006, p. 193 y ss; Séminaire La bête et le souverain, Volume II (2002-2003), Paris, Galilée, 2010. No entramos en ello.

3 «Le scandalon, qui est toujours de pierre et dit toujours la chute, sinon la chute de pierres...» Derrida, J., «Lignées», en Micaela Henich, Mille e tre, cinq, Bordeaux, William Blake \& Co., 1996, $\S 912$.
} 
do, «presque-trop», debieran, hubieran debido, dejar de sentir indiferencia justo antes de entrar en el proceso sin fin de su hipócrita melancolía. No obstante, situados siempre en el «pas au-delà» de la antiquísima sabiduría mineral ${ }^{4}$, los antropólogos filosóficos (para andarnos sin demasiados rodeos, los filósofos en general si empezamos a contar desde Kant), se frotan las manos con entusiasmo cuando les hacen salir de casa para hacer estudios de campo sobre las tonterías o las necedades, sobre las gansadas o las bobadas, sobre las sandeces, las majaderías, o, por decirlo de la manera más simple posible, sobre las mentecatas (mente captus) simplezas de los humanos. Ellos saben (demasiado bien) que, pese a todo, con la «bêtise», con el «événement-bêtise», ha llegado su momento: se lo van a pasar en grande, van a echar unas buenas risas. Y aunque quedarse de piedra sea a priori la condición sine qua non, la condición de posibilidad, de sus salidas de tono y de su sentido pseudo-hiperbólico del viaje, de la aventura o de la errancia (Like a Rolling stone ${ }^{5}$ ), aunque «quedarse de piedra» sea una y otra vez el punto de partida («la piedra de toque») y el de llegada («la piedra filosofal») de todos sus despropósitos, ellos, buenos amigos de lo imposible, aprovechan la oportunidad que les brinda su profesión para, poniéndose en marcha hacia lo colosal, hacia lo «sublime dinámico de la naturaleza», hacia la erección sin paliativos del que será, irremediablemente, el difunto falo ${ }^{6}$, hacer como si después de todo, paralizados como quedarán aún y siempre, una y otra vez, ante la mirada, el mal de ojo, de una inmortal Medusa, no pasara nada grave, nada aparentemente malo: «menos da una piedra», dicen; y justo a continuación, «Tous pour un! Un pour tous!» (embadurnados en un precario vitalismo auto-afectivo que todo lo baña, convocando jubilosos ante el tribunal de la razón la enésima cruzada, la penúltima operación-triunfo, contra la «bêtise»): «¡Es tiempo de hacerse el listo!, ¡La bêtise n'est pas mon fort! ${ }^{7}$ iSapere aude!».

Por lo demás, a pesar de precipitarse como se precipitan, hasta el fin, para acabar siendo los responsables de todas las bestialidades habidas y por haber, a pesar de ser necesariamente poco juiciosos la mayor parte de las veces para poder volver-

\footnotetext{
4 Que empiedra, en la herencia de Mallarmé y Lautréamont, la poesía francesa de posguerra (entre otros: R. Char, E. Gillevic, Y. Bonnefoy, A. du Bouchet, J. Dupin...). Cfr., Gourio, A., Chants de pierres, Grenoble, Ellug, 2005.

5 Que, pensará Ponge, no es una cosa fácil de definir correctamente. Ponge asegura que deberíamos de remontarnos a los tiempos anteriores al diluvio, dicho de otro modo, a la manera de Hegel, hasta antes de la religión de las flores y de la religión de los animales, de la ley del viviente y de los vivientes ejemplares, siguiendo la resonancia (Klang) de un enorme bloque pétreo, antepasado fabuloso e inocente de todas los cantos rodados. Cfr., Ponge, F., «Le Galet», en Le parti pris des choses, Paris, Gallimard, 1942. Igualmente: Cfr., Derrida, J., Glas, Paris, Gallimard, 1972, pp. 8a, 46a, 272-173a, 280-281a; La dissémination, Paris, Seuil, 1972, pp. 197, 398. El proyecto para los jardines (sin vegetación, de piedra y agua) del parque de La Villete, podría llegar a ser un buen ejemplo de todo esto. 6 Derrida, J., Points de suspension, Paris, Galilée, 1992, p. 53. Dicho de otro modo: «L'érection tombe». Derrida, J., Éperons. Les styles de Nietzsche, Paris, Flammarion, 1978, p. 84.

7 Valéry, P., Monsieur Teste, en CEuvres, vol. II, Paris, Gallimard, 1960, p. 15.
} 
se enciclopédicos, si algo tienen los antropólogos filosóficos, los filósofos en general o la sociedad de amigos de $S A^{8}$ (con Victor Cousin a la cabeza ${ }^{9}$ ), es conciencia, conciencia desgraciada y secreta, de la situación en la que se encuentran frente al objeto de su saber y curiosidad. A ellos no se les puede acusar de hacer las cosas a tontas y a locas: saben bien (por experiencia, dicen también) que salir de casa para recoger datos sobre la tontería en general (la bêtise en général) no deja de ser un pleonasmo más o menos ditirámbico: no dejan de trazar una y otra vez el mismo círculo. Caminar en círculo, ir dando tumbos, trazar un círculo vicioso, morderse la cola como las pescadillas... es otra manera de llamar a la «bêtise», una forma de hacer el tonto, de perder el tiempo, de mantenerse en el «pas au-delà» del meduseo «die Ewige Wiederkunft des Gleichen» ${ }^{10}$. Esto tiene al menos dos motivos bastante evidentes: en primer lugar, la tontería (bêtise), a pesar de su imantación apocalíptica, siempre se resiste, en última instancia, al columbarium, es decir, a las ordenanzas de la entomología, a la concluyente clasificación final, a la catástrofe ( $\alpha \tau \alpha \sigma \tau \rho o \varphi \eta ́)$ total; y en segundo lugar, el ojo de antropólogo filosófico se siente siempre y necesariamente como en casa cuando se trata de hacer estudios de campo en torno a la tontería o la necedad humana en general: la «bêtise» está envestida por $s u$ aire de familia, constituye, allí y donde acontezca, «la ley de la casa» (оікомонí): «loi de l'oikos (Heimlichkeit-Unheimlichkeit)»11. No obstante, cosa muy común, nunca hay tontería mayor, tontería más monumental, tontería más colosal, que comportarse como en casa cuando uno está fuera de casa: eso es tocar fondo originario (Urgrund); o mejor: ir a tocar originariamente fondo y encontrar-

8 «À la limite il pourrait y avoir de la bêtise dans le savoir absolu, en particulier, c'est l'exemple auquel pensent et que donnent tous ceux qui parlent de la bêtise...» Derrida, J., Séminaire La bête et le souverain. Volume I (2001-2002), Paris, Galilée, 2008, p. 203.

9 Victor Cousin: «philosophe très français», dice J. D., traductor de Platón y de Hegel al alemán, al que Flaubert se refiere en su correspondencia como «le Philosophe». J. D. habla de Cousin y de Hegel en torno a la « bêtise» en «Une idée de Flaubert: 'la lettre de Platon'», texto que trataremos muy brevemente más adelante. Sobre el ansia de Cousin de hegelianizar Francia convirtiendo a los franceses en una sapientísima bandada de aquilinos ya da cuenta J. D. en Glas («Hegel, dites-moi la vérité, puis j'en passerai à mon pays ce qu'il pourra comprendre...» Cfr., Glas, op. cit., p. 208a y ss.) y también en «Lemmes» (Cfr., «Lemmes», en La vérité en peinture, Paris, Flammarion, 1978, p. 23).

10 Como es sabido, Nietzsche establece una analogía entre el eterno retorno de lo similar y la cabeza de Medusa: «der große Gedanke als Medusenhaupt». Cfr., Nietzsche, F.,. Nachlaß 1884-1885, en KSA, bd. 11, München, Deutscher Taschenbuch Verlag, 1999, p. 360. En relación con el fetiche y la castración a propósito de la Medusa en Nietzsche: cfr., Pautrat, B., «Nietzsche médusé», en Nietzsche aujourd'hui?, vol. I. Intensités, Paris, 1973, pp. 9-30. J. D. habla de la parálisis y del pensamiento de la Medusa a propósito de M. Blanchot en «Pas». Cfr., Derrida, J., «Pas», en Parages, Paris, Galilée, 2003, p. 76. En relación con la Medusa y la castración vía Freud (Das Medusenhaupt, 1922) en J. D.: cfr., «Hors livre», en La dissémination, op. cit., p. 47-48 (en donde se citan también un montón de piedras a propósito de Lautréamont y de su proceso de cretinización del lector); Séminaire La bête et le souverain. Volume I (2001-2002), op. cit., p. 298 y ss.

11 Derrida, J., «Survivre», en Parages, op. cit., p. 113. 
se con que no lo hay (Ungrund): tocar fondo abismalmente (Abgrund) y descubrir, por enésima vez, el territorio ultra-filosófico en el que se da toda «bêtise». Pequeña historia de contradicciones que los antropólogos filosóficos, gente seria y necesariamente aburrida por lo general, saben que hay que saber llevar para no tirarse piedras en su propio tejado: al fin y al cabo, dicen, en ello se juega finalmente su profesión y profesionalidad.

Por todo ello, al menos, en lo que hace a su auto-consciencia declarada y triunfal, desde los tiempos de la Kritik der Urteilskraft, en un funerario petroglifo informe, pseudo-sepultura cuasi-críptica, memoria mineral ${ }^{12}$ de lo monumental situado a las afueras de sus conciencias ${ }^{13}$ cuya caída («précipice de la pensée» ${ }^{14}$ ) esperan tras el día del Juicio, tras el gran seísmo, la vulcanización, la carbonización definitiva de los nombres propios ${ }^{15}$, los filósofos-litógrafos, puliendo previamente la superficie con una hermosa piedra pómez ${ }^{16}$, asumiendo una y otra vez el papel de Perseo (en el papel a su vez de Forrest: «tonto es el que hace tonterías») y haciendo constantemente como si hubieran decapitado, como si decapitasen una y otra vez, a Medusa (en el papel a su vez de Jenny: «itu eres tonto o te lo haces?»), han sublimado su constituyente «bêtise» grabando con un bonito cuchillo pedernal ${ }^{17}$ las siguientes palabras: «Ecce homo: wie man wird, was man ist ${ }^{18}$. Ellos, que saben poco de piedras y menos, por lo tanto, de la erosión que se come las pirámides ${ }^{19}$, creen que durará para siempre, no prestan atención a «l'imminence du péril concentre nos regards [...] sur la pierre où se résument sa possibilité et sa fragilité» 20.

Si nos dejamos pues aquí llevar un poco por «el aire de imbecilidad» que alimenta las proclamas y los cánticos de libertad de los antropólogos filosóficos y suponemos, en consecuencia, el asesinato por poco ejecutable que el mismo sea, si hacemos como si efectivamente, los filósofos perseo-edípicos amigos de $S A$, estuviesen detrás, una y otra vez, con cada vocablo que pronunciasen, de manera no eterna pero sí indefinida, de la supuesta cabeza rodante de una Gorgona ejecutada cruelmente por decapitación, si suponemos para colmo que ellos, titiriteros trascendentales de poca monta, saben, mezquinamente y/o tontamente, utilizar la cabeza,

12 Cfr., Derrida, J., Demeure, Athènes, Paris, Galilée, 2009, p. 27.

13 Derrida, J., Glas, op. cit., p. 288a.

14 Derrida, J., «Lignées», op. cit., §815.

15 Ibíd., $\$ 810$.

16 Cfr., Derrida, J., Signéponge, Paris, Seuil, 1988, p. 91 y ss.

17 Derrida, J., Circonfession, en Derrida, J., \& Bennington, G., Derrida, Paris, Seuil, 1991, pp. 65, 103.

18 A propósito de la tontería Nietzsche se hace también un poco el listo siguiendo la estirpe perseoedípica, es decir, siguiendo, como hace de vez en cuando, a su padre. Cfr., Nietzsche, F., Ecce homo. Wie man wird, was man ist, en KSA, bd. 6, op. cit., p. 271.

19 Derrida, J., «La différance», en Marges de la philosophie, Paris, Minuit, 1972, pp. 4, 25; «Le puits et la pyramide», en ibíd., pp. 95, 115-116. Glas, op. cit., p. 188a.

20 Derrida, J., «Force et signification», en L'écriture et la différence, Paris, Seuil, 1967, p. 13. 
su poder infeccioso, en su propio provecho dejando al resto de piedra (literalmente pasmados) ${ }^{21}$, supongamos en fin, imaginémoslo aunque con ello no vayamos a ningún lado, el sempiterno Juicio y la lapidaria sentencia que precedió, que precede una y otra vez, a la caída de una lápida sepulcral que selló, que sella continuamente, mitigando sus miedos, la condena a muerte de unos antropólogos filosóficos finalmente ejecutados por lapidación a cuenta no ya tanto de su incorregible «bêtise» sino de su no haber aprendido a vivir con ella22 (no haber querido comprender que la misma «est au cour de la philosophie»), a causa en fin de ser tontos de remate, de su soberana y estrictamente apocalíptica «bestialité». Si algo es propio de los antropólogos filosóficos, de los filósofos y de los hombres como tales, es que nunca aprenderán (a morir, porque de ello se trata): chocarán siempre, una y otra vez, de manera escandalosa, con la misma piedra. Por ello mismo, cuando se trata de ellos, de su incomunicación constitutiva: «toda la comunidad hablaba de apedrearlos» (Números, 14, 10)23. Así pues: «Devenir-bête», «devenir-pierre»: «dumb as a sack of rocks» cuando uno se cree precisamente de piedra, poderosamente pétreo, no ya Iron, sino Stoneman, Stone-phalus-man, es decir, cuando uno se comporta como si fuera supuestamente invulnerable, imperturbable, eterno, no-biodegradable.

Debemos pues ahora, antes de que sea demasiado tarde y se levante el luto tras su inhumación, aprovechar la oportunidad: «la pierre résonne et vibre encore, elle émet une sorte de jouissance douloureuse et indéchiffrable...»24, «on aura dit qu'elles formaient les tableaux en ruines d'une histoire disparue et que l'air lui-même avait incisé la pierre pour y déposer les pensées de la pierre que la pierre ne pouvait pas voir» ${ }^{25}$, todavía «...restent les interstices d'une archive pierreuse, autrement dit sans éloquence» 26 en «le vieux cimentière juif de Prague. Pierres à la fois plantées et dressées de cris poussés vers le ciel» ${ }^{27}$. [Quede pues aquí expuesto el contenido de la sentencia de manera telegráfica, ultra-esquemática, ultra-kantiana. Partiremos del contexto de su última aplicación, es decir, del momento en el que J.

21 Como es sabido, J. D. cita al final de la sexta sesión del primer volumen del seminario unas palabras de Celan que mencionan a Medusa en un escenario de marionetas: «uno quisiera ser una cabeza de Medusa, para poder convertir en piedra a un grupo así, y llamar a las gentes». Algo más adelante: «uno quisiera ser una cabeza de Medusa, para... jaferrar lo natural como lo natural por medio del arte! Cfr., Celan, P., Der Meridian, cit. por J. D., Séminaire La bête et le souverain. Volume I, op. cit., p. 251 y ss.

22 «J'ai fait une bêtise, cela ne veut pas dire que je suis bête mais au contraire: alors même que je ne suis pas supposé être bête, je me suis laissé aller, surprendre à faire une bêtise». Derrida, J., Séminaire La bête et le souverain. Volume I (2001-2002), op. cit., p. 205.

23 Cfr., Derrida, J., Mal d'archive. Une impression freudienne, Paris, Galilée, p. 104.

24 Derrida, J., Points de suspension, op. cit., p. 128.

25 Sollers, P., Nombres, Paris, Gallimard, 1968, p. 40 (citado por J. D. en «La dissémination», en La dissémination, op. cit., p.402).

26 Derrida, J., «Lignées», op. cit., $\$ 872$.

27 Ibíd., $\$ 836$. 
D., todavía «au borde du cadavre de Carolina, la mère et homonyme de Loulou», siguiendo la pista de Flaubert desde la memoria de Pierre Bourdieu y en el escenario de su último seminario, en la antesala, eterna, de lo que es una deconstrucción de la muerte 28 , de la suya propia, comienza a abandonar, sabiéndose, desde siempre, infectado ya, desde el origen, por los ojos de Medusa].

«Ce petit traité de la bêtise». Son las palabras con las que J. D. comienza a despedirse de los asistentes en la doceava sesión del primer volumen de su seminario La bête et le souverain (2001-2002): «Abandonnons ici ce petit traité de la bêtise». Parece interesante señalar que, después de haber hablado de la «bêtise» largo y tendido - a partir principalmente de Deleuze ${ }^{29}$, de Ronell30, de Valéry y, en cierto modo, de manera oblicua, de Celan (sesiones V a VIII principalmente) -, la despedida se pone en marcha justo después de: por un lado, de manera ciertamente condescendiente, decir que encuentra a Heidegger un poco «eigensinnig», un poco «bête lui-même» (sabemos que Heidegger reconoció, J. D. y Ronell insisten en ello, haber hecho una tontería [Dummheit], al menos una, y no simplemente haber cometido un error...) a propósito de ciertas decisiones sobre la vida-la muerte; y por otro, justo después de señalar, de nuevo, en una especie de cancioncilla cáustica que prende el volumen de cabo a rabo («chaque fois l'auteur de Homo sacer serait le premier à dire qui aura été le premier...»), algunos olvidos y descuidos, algunas «bêtises» filológicas de diverso grado desperdigadas por los escritos de Agamben a propósito igualmente, en última instancia, de ciertas convicciones sobre la vida-la

28 Cfr., Derrida, J., Séminaire La bête et le souverain. Volume II, op. cit., p. 234.

29 Unas cuentas consideraciones a quemarropa: sin duda puede llegar a ser motivo de sorpresa, quizá lo deba llegar a ser, que algunas de las pocas páginas que J. D. le dedica a Deleuze tengan que ver con la «bêtise». A pesar de reconocerle numerosas cosas en lo que hace a este problema (la «bêtise» como algo que estaría más allá del conocimiento, la relación establecida por Deleuze entre la «bêtise» y el mal, entre la «bêtise» y la soberanía), la operación que J. D. realiza finalmente con Deleuze es similar a la que realiza respecto a Flaubert y Baudelaire. Pareciera como si para J. D., Deleuze, en última instancia — dejándose llevar por la velocidad o el sarcasmo, dejándose llevar por ese sentimiento de «ver la 'bêtise' y no poderla tolerar», considerando igualmente que la misma se daría exclusivamente en el hombre (que los pobres animales, prevenidos como están del fondo, no podrían hacer nunca tonterías)- también, como Flaubert o Baudelaire, se hubiera pasado de listo. La «bêtise» hay que poderla tolerar (tolerar lo intolerable) porque precisamente, tal y como dice Deleuze, es siempre la mía («...la bêtise n'est jamais celle d'autrui, mais l'objet d'une question proprement transcendantale: comment la bêtise (et non l'erreur) est-elle possible». Cfr., Deleuze, G., Différence et répétition, Paris, Puf, 2003, p. 197).

30 Ronell habla de la «stupidity» como «cuasi-concepto»: inestable, variable, plástico, móvil: «...it remains to be seen whether stupidity can be viewed as a concept». (Cfr., Derrida, J., Séminaire La bête et le souverain. Volume I (2001-2002), op. cit., p. 231 y ss; Ronell, A., Stupidity, Urbana \& Chicago, University of Illinois Press, 2001, p. 68). No entramos en ello. 
muerte: «Abandonnons ici ce petit traité de la bêtise, d'un bêtise qui peut-être revient toujours à s'en tenir à quelque opinion opiniâtre sur la vie la mort ${ }^{31}{ }_{\gg}$. No obstante, no vamos a entrar aquí ni en lo que serían las gansadas de Agamben ni en lo que podrían ser las burradas de Heidegger: ni uno ni otro se habrían dado cuenta de que «il y a un certain Triumph of Life dans la bêtise» ${ }^{32}$. Tampoco vamos a realizar un análisis de la problemática desarrollada en las sesiones anteriores a partir de los autores citados. En lo que vamos simplemente a insistir es en que, repetimos, llegado el momento J. D. «abandona» («Abandonnons ici...»), comienza a abandonar, no tiene la menor intención de concluir, su tratadito sobre la tontería a fin de cuentas su pequeño tratado de antropología filosófica si aceptamos que la «bêtise» es «lo propio del hombre», o más exactamente, si estamos convencidos de que la «bêtise» es «le propre du propre» (del hombre)-. Así pues, ni acaba ni concluye, sino que, a pesar de aseverar, mediante la poco reveladora, la cuasi-apocalíptica tonalidad que le es característica, que «the end is coming» ( «La fin est proche, la fin, veux-je dire, du séminaire de cette année»), asegura que permanecerá finalmente, decididamente, como siempre a fin de cuentas, sobre el umbral («nous sommes toujours sur le seuil»). De hecho, si J. D. encuentra a Heidegger un tanto tontito, y si se ha encargado de recoger unas cuantas tonterías de diverso grado dispersas por los escritos de un buen sabueso como Agamben-rastreador-de-principios, es precisamente porque ellos se han resistido a abandonar, porque no han querido permanecer en el umbral, es decir, porque no han podido resistirse a concluir, porque, en definitiva, han actuado como buenos antropólogos filosóficos: emitiendo «veredictus». «Querer concluir», no saber abandonar, haber aprendido a hacerse de tanto en tanto un poco el tonto o el longuis respecto a ciertas urgencias para llegar a donde tenían que llegar: ahí encontramos al antropólogo filosófico, he ahí el gesto mismo del filósofo, del filósofo en cuanto tal, del filósofo propiamente dicho, es decir, del filósofo trascendental: el gesto de su soberana majestad. Pero evidentemente, y de esto se trata, he ahí, al unísono, el momento en el que acontece no ya simplemente su «bêtise» («Oui, la bêtise consiste à vouloir conclure»), sino su ser-«bête», el instante mismo del envío de las piedras ${ }^{33}$, del apedreamiento, la hora de su condena pública a muerte por lapidación: priapismo soberano, erección auto-inmunitaria: «bêtise ithyphallique», dirá J. D. a cuenta de una triste historia de falos petrificados ${ }^{34}$. Así pues: ¿de dónde viene este querer concluir (una modalidad más del querer-decir, es decir, de la «Bedeutung» que inquietaba desde los primeros momentos

\footnotetext{
31 Derrida, J., Séminaire La bête et le souverain. Volume I (2001-2002), op. cit., p. 410.

32 Ibíd., p. 249. Como es sabido, en Survivre, J. D. comenta el poema de Shelley The Triumph of Life en el horizonte de L'Arrêt de mort de M. Blanchot.

33 «Les collectionneurs de pierres ne peuvent communiquer entre eux en s'envoyant la pierre». Derrida, J., «Envois», en La carte postale. De Socrate à Freud et au-delà, Paris, Flammarion, 1980, p. 222.
} 
el trabajo de J. D. desde la recitación del «I am dead» de Poe en el corazón de la fenomenología de Husserl) y de dónde vienen las cientos de piedras que lo acompañan?

Ciertamente, uno podría pensar que después de todo, piedra sobre piedra, a lo tonto a lo tonto, abandonando finalmente el asunto así sin más, J. D. no habría definido a lo largo de su tratado sobre la «bêtise» la «bêtise» misma, no se habría hecho cargo de qué sea la «bêtise» (Qu'est-ce que...la bêtise?) como tal, no habría sido fiel (la tentación de San Antonio) al deseo de «voir la bêtise elle-même». Ahora bien: si esto es así es porque J. D. no es ciertamente ningún tonto. Pese lo que le pese a la madre de Forrest, ciertamente tonto no es, en la exigencia de performatividad total que exigiría, el que hace tonterías (stupid is as stupid does). J. D. habría sido tonto, el más tonto de la clase, tonto de capirote con sambenito incluido, habría sido de hecho un auténtico bestia, si, después de haber mareado tanto la perdiz (de Deleuze a Valéry pasando por Ronell y desviándose hacia tantos otros), después de haber andado en círculos más allá de todo vicio (andaduras, estrategias de viejo cazador, que J. D. hiperbolizará en el segundo tomo de este mismo seminario siguiendo una pista milenaria), hubiera acabado, soberanamente, definiendo la tontería, es decir, diciendo que jamás ha cometido tontería alguna o emprendiéndola contra la tontería: ese sería el modo de ser rematadamente tonto («bête à mourir»), aunque «rien n'est plus bête que l'intelligence même de ce désir», simplemente la manera de ser un auténtico petardo. Dicho de otro modo, el modo que tendría una voluntad infinita de imponerse (Brecht), precipitadamente, a un entendimiento finito 35 : la definición implicaría la localización de un sentido fundador y el mismo la posibilidad de una traducción sin resto. Ahora bien: si la definición es la conclusión misma, hay que decir que entonces las conclusiones son las bestialidades mismas derivadas de su necio afán: acabar con, concluir de una vez por todas. Por lo tanto, a pesar o por la razón misma de que J. D. ha dedicado todo un año a la «bêtise», J. D. no acaba con la «bêtise»; la «bêtise» finalmente, se abandona a sí misma... «Il conclut (bêtement, donc) qu'il ne veut pas conclure (ce sera trop bête). Il le faut d'un " $n i . . . n i$ " ${ }^{36}$. [Todo esto viene efectivamente de lejos. Y en ello es en lo que vamos a insistir finalmente para intentar rastrear dos cabos sueltos desde los que parece nacer esa sentencia de muerte que dice que la «bêtise» consiste, simplemente, tontamente, en querer concluir. Los mismos cabos cuelgan a su vez dos nombres propios: Flaubert y Baudelaire. Aquí no pretendemos atarlos, aunque sí encadenar-

\footnotetext{
34 «Pierre est le phallus». Derrida, J., «Hors livre», op. cit., p. 47. Cfr., Séminaire La bête et le souverain. Volume I (2001-2002), op. cit., p. 204 ; «Le phallos est, lui-même, comme la chose même qu'il est, une sorte de marionnette». Ibíd., p. 296.

35 Derrida, J., Séminaire La bête et le souverain. Volume I (2001-2002), op. cit., p. 204.

36 Derrida, J., «Une idée de Flaubert: 'la lettre de Platon'», en Psyché. Inventions de l'autre, Paris, Galilée, 1987, p. 315.
} 
los a propósito de una serie de cuestiones].

Primer cabo suelto: no hemos dejado de subrayar que lo pétreo, lo pedregoso, no está pues nunca lejos de la «bêtise», y que no estándolo, la «bêtise», a su vez, es lo propio de la filosofía en la medida en que es lo propio del hombre. No sabemos ciertamente qué es la «bêtise», aunque sepamos que consiste en querer concluir. Tampoco sabemos qué es una piedra — recordemos que J. D., a cuenta de ciertas inscripciones psicoanalíticas que irían desde Freud («Freud et la scène de l'écriture»), hasta Abraham/Torok («Fors»), «no quería presuponer el saber de lo que es una piedra» 37 - De todos modos, al margen de no saber qué son, «sans savoir ce qu'on veut dire»: ¿qué hace J. D. con las piedras y con las tonterías? ¿qué relación hay entre la tontería, las piedras, particularmente las preciosas, y... el mal, el mal radical?

«Oui, la bêtise consiste à vouloir conclure» ${ }^{38}$ : indeterminación de la «bêtise» (Ronell) más allá de la idealidad sin rozamiento de su sentido como indeterminación. J. D. encuentra esta sentencia de Flaubert en su correspondencia. A la misma le adjunta otra no menos importante: «La bêtise est quelque chose d'inébranlable; rien ne l'attaque sans se briser contre elle. Elle est de la nature du granit, dure et résistante» 39 . Trabajará sobre estas dos afirmaciones particularmente en «Une idée de Flaubert: 'la lettre de Platon'» (1980), conferencia (pronunciada en un congreso celebrado en el centenario de la muerte de Flaubert) en la que debían establecerse las relaciones de Flaubert con la filosofía. Años más tarde, en la sesión quinta de su seminario La bête et le souverain (2001-2002), retomará algunas de las cuestiones tratadas a lo largo de la conferencia de 1980 desviando las mismas hacia otros lugares. Mientras que la primera de las afirmaciones está sacada de una carta que Flaubert le dirige a Bouilhet el 4 de septiembre de 1850, la segunda la encontramos en otra que le envía a Derain en octubre de 1850. Ni temática ni cronológicamente están lejos la una de la otra. Lo determinante es que, a la segunda de las afirmaciones, citada en nota a pie, la acompañan unas consideraciones de Flaubert sobre lo

\footnotetext{
37 Derrida, J., «Moi - la psychanalyse», en Psyché Inventions de l'autre, op. cit., p. 158. Antes hemos recogido la frase: «Pierre est le phallus». No obstante, Derrida se preguntaba a continuación: «Est-ce une réponse?» Derrida, J., «Hors livre», op. cit., p. 47. Igualmente: «Et si c'était la bêtise même, le phallus?» Derrida, J., Séminaire La bête et le souverain. Volume I (2001-2002), op. cit., p. 345.

${ }^{38}$ Flaubert, G., Carta «a Louis Bouilhet. 4 septembre 1850», en Correspondance, I, 1830-1851, Paris, Gallimard, 1973, p. 679-680, citado por J. D., en «Une idée de Flaubert...», op. cit., p. 309; Séminaire La bête et le souverain. Volume I (2001-2002), op. cit., 220.

39 Flaubert, G., Carta «a Parain, 6 Octobre 1850», en Correspondance, vol. I, op. cit., p. 689, cit. por J. D., en «Une idée de Flaubert...», op. cit., p. 315; Séminaire La bête et le souverain. Volume I (20012002), op. cit., 219).
} 
tremendamente cretinos que son aquellos individuos que inscriben sobre las piedras su nombre (para devenir, uno lo supone, invulnerables: maneras de querer eternizarse...: «pour Flaubert la bêtise est toujours monumentale, de la taille du monument pierreux couvert d'inscriptions»). Dirá J. D. al final de la nota, tras citar el extracto de la carta, que: «Le nom propre s'y incorpore et ce n'est pas un bénéfice secondaire de cette spéculation sur la bêtise. J'avais commencé par souligner les pierres».

En efecto, J. D. había comenzado recalcando las piedras. Antes de que apareciera en escena la «bêtise», antes de citar las afirmaciones de Flaubert sobre esa «bêtise» que ejerce sobre él - amante como es de la filosofía y de las ideas (palabra sobre la que girará toda la conferencia en relación con la herencia platónico-hegeliana)_, una «fascinación propiamente diabólica», antes de entretenerse con los problemas de su contagio (a propósito de Bouvard et Pécuchet) y de establecer las relaciones, sin duda problemáticas, que mantendría Flaubert con la filosofía, J. D. había comenzado destacando unas piedras justo en el momento en que recogía otras dos cartas más: una, la primera de todas ellas, de 1868, en la que Flaubert le decía a su sobrina Loulou, huérfana pero amante del ideal, que leyese el Banquete y el Fedón (en la traducción de Cousin), y otra, la segunda, de 1846, año de la muerte de su hermana (madre de Loulou), en la que le decía a su amante Louise Coulet (recientemente separada de Cousin): «Tu donnerais de l'amour à un mort. Comment veux-tu que je ne t'aime pas? Tu as un pouvoir de attraction à faire dresser les pierres à ta voix...». Estas últimas son pues las piedras que comienza señalando J. D. antes de citar las palabras sobre el carácter pétreo de la «bêtise». Ahora bien: ¿de qué se trata con todo ello? ¿Qué dicen esas piedras? La muerte, sin duda... dicen y hacen la muerte, pero quizá también una cierta manera de vivir, de aprender a vivir, con ella.

Al final de la conferencia J. D. escribirá lo siguiente: «il fallait aussi faire justice à une phrase [de Flaubert], une seule, que j'aurais voulu inscrire sur la pierre de tout ce qui se méduse au bord du cadavre de Caroline, la mère et homonyme de Loulou - c'est pourquoi j'ai préparé la pierre depuis une demi-heure, celle sur laquelle on a toujours la bêtise de graver un nom, la 'colonne" de granit, dure et résistante' $\gg 40$. La frase, que J. D. habría querido inscribir sobre la piedra, es la siguiente: «...acharne-toi sur une idée! ces femmes-là au moins ne meurent pas et ne se trompent pas!». Está sacada de una carta que Flaubert le envía Feydeau (1215 de noviembre de 1859). La misma no diría nada, o al menos no demasiado, si no fuese porque J. D. parece que pretende, citándola al final, confirmar el duelo imposible de Flaubert respecto a su hermana muerta y, a su vez, no menos una cosa que la otra, la responsabilidad infinita del propio Flaubert respecto al porvenir de su sobrina (de la que se ha hecho cargo). Duelo y responsabilidad ante un único nom-

40 Derrida, J., «Une idée de Flaubert...», op. cit., p. 325. Subrayado nuestro. 
bre desdoblado: («Caroline», nombre de la hermana y de la sobrina, de la madre y de la hija), en un decorado compuesto a base de piedras de principio a fin.

En efecto, la operación parece ser la siguiente: J. D., antes de citar esa frase que, repetimos, él habría querido inscribir para hacerle justicia (a la propia frase y a Flaubert), recupera otra escena de correspondencias. Esta vez la escena la componen las frases de un Flaubert desconsolado que, entre gemidos, balbuceos y lágrimas - amando la idea de la «nada absoluta» como la ama, mostrándose desconsoladamente spinozista - intenta digerir, en un doloroso duelo, la muerte de su hermana («lo imposible», escribirá J. D.). Las frases se las escribe a M. Du Camp (2223 de marzo de 1846) desde la misma habitación en la que se encuentra su hermana muerta: en ellas dice que su madre es una estatua que llora, confiesa tener los ojos secos como el mármol y haberse quedado también seco como la piedra de una tumba, sin vida, más muerto que vivo. Creemos que en esta escena se pondría en marcha la naturaleza secreta, sin concesiones, de la piedra, de lo pétreo-mortal, en su relación con la «bêtise», es decir, con el querer-concluir, reconociéndose, más allá de la arrogancia, de la vanagloria, y del régimen de la acusación petulante (propia de los filósofos que él, a su modo, también representa), más allá del acto bélico («duellum») o de la maniobra aristocrática que tendría como misión la elevación moral de una nación o, peor aún, la huída (escena ejemplar del Arca que recogíamos al comienzo como manifestación por antonomasia del «stupid one», precisamente, del arca de la jactancia como arca de la nada - «der Schrein des Nichts»-), un haberse quedado sinceramente, vulnerablemente, de piedra («dŏlus»). Ahora bien: este es (en el umbral de la penúltima escena) el lugar en el que se hace finalmente comparecer a su vez a la idea (a Loulou, la idea del propio Flaubert) y al ideal enviados a un Feydeau endeudado. Si Loulou (la idea, una, la de Flaubert dirá J. D.) se ha quedado huérfana, ella debiera seguir amando el ideal como lo ama (lecturas de Platón...): las que lo aman, las que se agarran a una idea más allá de toda determinación, son las mujeres que no mueren, en cualquier caso, las que no quieren morir, las que están colocadas en el «au-delà» de un cierto «Todestrieb», las mujeres que no «devienen-materia» (Sartre), que no se vuelven tontas, tontas como piedras... «...acharne-toi sur une idée! ces femmes-là au moins ne meurent pas et ne se trompent pas!».

Segundo cabo suelto: al menos ya a partir de Donner le temps (y resulta provechoso poner en el horizonte el espacio dedicado a Lacan en L'animal que donc je suis, cuyo núcleo se repetirá en la cuarta sesión del primer volumen de La bête te le souverain, precisamente en la antesala del tratamiento de la «bêtise»), uno podría preguntarse también cosas como las siguientes: ¿A cuánto está, a cuánto se sitúa, la 
bestialidad de la tontería? ¿Qué tontea, en sentido estricto, con qué? ¿Cuáles son los pasos a seguir para devenir-bestia desde un devenir-tonto? ¿Qué se da antes: la bestialidad o la tontería? ¿Se siguen una de la otra? ¿operan mediante entrecruzamientos parasitarios? La «bêtise», cuando aparece en este texto de 1977-78, es decir, cuando aparece para ser tratada como tal, es en relación con el mal y la crueldad: «Le plus irréparable des vices est de faire le mal par bêtise» ${ }^{41}$. La afirmación se encuentra en el final del pequeño poema en prosa de Baudelaire que J. D. comenta en Donner le temps: «La fausse monnaie». Pese a ser puesta en boca de Baudelaire, ese mal, el más irreparable de los vicios, el vicio imperdonable, tiene como referencia inmediata a Sade, al ultrakantismo holbachiano de Sade. Justo a continuación, en una nota al pie J. D. reproduce la seriación «bêtise diabolique/cruauté satanique (bestialité)》 desviándola hacia algunas consideraciones de Kant sobre el mal radical repartidas por Die Religion innerhalb der Grenzen der blossen Vernunft. No entramos en ello tampoco. De alguna manera, lo que nos interesa es ver cómo en el querer concluir como propio de la filosofía que constituye la gran sentencia de muerte de la misma filosofia, en el querer concluir en general como propio de ese animal que tiende a fines conclusivos, terminales, que es el hombre, en el querer concluir como proceso de apropiación placentera, y en la «bêtise» como apropiación misma de ese proceso, ya está el mal injerto, el perjurio. Pero el mal radical (más allá de este mal constituyente que le es propio a la archi-caída), sería quizá ser verdaderamente «bête», y ser así, a la par, verdaderamente «bestial», matar, en cualquier caso creer que uno ha matado, mediante todas las luces, mediante todo el saber del mundo, definitivamente, en la explosión de una carcajada satánica (verdaderamente baudelaireana) que pondría en escena una superioridad ilimitada, al monstruo telúrico, a Medusa. Y ello no precisamente por «bêtise», sino por ausencia de ella: por pretender llevar el cálculo hasta el final. Más allá de la infinitud de la voluntad que caracteriza a la «bêtise» como precipitación aspirar pues a una infinitud del entendimiento que se sobrepusiese, que castrase, a la propia voluntad; es lo que exige, sádicamente, el sarcasmo final del narrador de «La fausse monnaie». Pensará el narrador: se le podía haber perdonado el desliz, pero no ha calculado bastante: ha cometido una tontería, se ha comportado de manera poco inteligente: y eso, precisamente eso, es lo imperdonable: no haber podido, no haber sabido, ni finalmente haber querido (y esto es lo más grave), comprender, comprender realmente el mal que iba a producir, lo malvado que era en el fondo. Saber, hacerse cargo de ello, pensará el narrador, hubiera al menos sido un mérito ( $O n$ n'est jamais excusable d'être méchant, mais il y a quelque mérite à savoir qu'on l'est; et le plus irréparable des vices est de faire le mal par bêtise»). No obstante, nos pre-

41 Baudelaire, C., «La fausse monnaie», en Cuvres complètes, vol. 1, Paris, Gallimard, p. 323. Cfr., Derrida, J., Donner le temps. 1. La fausse monnaie, Paris, Galilée, 1991, p. 49, 208 y ss. 
guntamos: ¿Es esto posible? ¿No es esta creencia, la de imaginar que uno puede y debe efectivamente concluir, precisamente la manera de volverse rematadamente tonto, peligrosamente bestia, irremediablemente estúpido por una supuesta facultad de elevación espiritual, de vuelo de albatros más allá de toda perversión y de todo satanismo? ¿No es este también el mal auto-inmunitario de Príamo?

«Contagion de la bêtise»... Definitivamente y con ello también nosotros abandonamos: no hay nada más tonto, más propiamente tonto, nada más natural, más propio, más común a todos los mortales (más allá de Heidegger, y del lado de las piedras que saben morir), que quedarse, de repente, de piedra, petrificado: sorprendido por una mirada medusea, declaradamente vulnerable: «La peur d'une méduse, la légitimité même» ${ }^{42}$. Se trata pues no ya tanto de decir «c'est bête, ce qui arrive» ${ }^{43}$ (cosa que podría ocurrir... uno sabe que esas cosas pasan, le pasan, continuamente) sino «c'est bête ce qui arrive»: y ese «bête», que tendría el poder petrificante de la «bêtise», irrumpiría como tal en el proceso mismo de toda apropiación, de manera necesaria, desafiando todo mal de archivo, toda aspiración a la conservación, a conservarse o a conservar, eternamente. Por nuestra parte, cuidémonos de hacer el tonto más de la cuenta, de extender más de la cuenta lo que no deja de ser una «longue narration pétroglyphique, une série ininterrompue d'histoires interrompues.... ${ }^{44}$, cuidémonos de saber qué es lo que realmente queremos decir porque esto de la tontería no es sin lugar a dudas ninguna tontería, no es cosa de poca monta: quién sabe, si la tontería es siempre una manera de no comprender, de no querer comprender, si la tontería es por principio mi fuerte, si marca la diferencia, si es cosa mía (¡dejádmela a mí!), váyase que por mal del demonio queramos comprender definitivamente, mantenernos imperturbablemente vectoriales, y entonces creer que podemos morir de «bêtise» 45 , tocar fondo cabezonamente, no saber-aprender a morir con la «bêtise» y cometer la bestialidad de pensar, en fin, que es posible suicidarse realmente. Por si las moscas: «Etcétera», líbrennos del formol, amén... y colóquese un «To be continued» o unos «points de suspension» detrás de esa «bêtise» y de esa «bestialité» que encadenan frívolamente el bello destino de los vivos, de todo lo que va errante como el plancton ( $\pi \lambda \alpha \gamma \kappa \tau o ́ v)$ - desde las tulipas salvajes hasta los unicornios imanta-vírgenes pasando por los erizos de carretera-, más allá de la pacífica sabiduría mineral de los cantos rodados y de la historia interminable de los comepiedras.

«Si bien que contrairement à l'opinion commune qui fait d'elle aux yeux des hommes un symbole de la durée et de l'impassibilité, l'on peut dire qu'en fait la pierre ne se

\footnotetext{
42 Derrida, J., L'archéologie du frivole, Paris, Galilée, 1990, p. 146.

43 Derrida, J., Séminaire La bête et le souverain. Volume I (2001-2002), op. cit., p. 193.

44 Derrida, J., «Lignées», op. cit., §912.

45 Cfr. Derrida, J., Apprendre à vivre enfin. Entretien avec Jean Birnbaum, Paris, Galilée, 2005.
} 
reformant pas dans la nature, elle est en réalité la seule chose qui y meure constamment».

F. Ponge, Le Galet. 